\title{
Phase Separation Mechanism of Agar-DMSO-EtOH Ternary System in the Process of Wet Spinning
}

\author{
Kang Yang ${ }^{1,3, a}$, Zhixin Xue ${ }^{1,3, b, *}$, Weiwei Zhang ${ }^{1,3}$, Yanzhi Xia $^{2,3}$ \\ ${ }^{1}$ School of Chemical Science and Engineering, Qingdao University, Shandong 266071, China \\ ${ }^{2}$ State Key Laboratory Base, Qingdao University, Shandong 266071, China \\ ${ }^{3}$ Biomass Fiber and Textiles Synergy Innovation Center, Qingdao University, Shandong 266071, \\ China \\ a24130151@qq.com, bxuezhixin92@163.com
}

Keywords: Agar, Phase separation, Cloud-point curve, Wet spinning.

\begin{abstract}
Phase separation have been measured at the temperature of $25^{\circ} \mathrm{C}, 45^{\circ} \mathrm{C}$ and $65^{\circ} \mathrm{C}$ for the polymer (agar) + solvent (DMSO) + no solvent (ethanol) ternary system by using a titration method. Through combining experimental data and empirical equations, ternary phase diagram was drawn to illustrate the mechanism of spinning. Low temperature was beneficial to produce fibers and instead of ethyl alcohol with water as coagulation bath is feasible, which were shown clearly in the ternary phase diagrams.
\end{abstract}

\section{Introduction}

Agar is a phycocolloid extracted from the cell walls of a class of red algae (Rhodophyceae), including Gelidium, Gracilaria, and Porphyran spp. As a biological material, agar has good biocompatibility, biodegradability and excellent moisturizing capacity. Therefore it has been widely used in the fields of food and medicine.

Agar belongs to the galactan family and is a complex polysaccharid essentially composed of hybrid structures alternating (3-O)-linked $\beta$-D-galactopyranose and (4-O)-linked $\alpha$-Lgalactopyranose. Agarose is the main ingredient in agar. The main repeating moiety in agarose is 3,6-anhydro- $\alpha$-L-galactose with few variations and low content of sulfate esters, for a molecular weight of $306 \mathrm{~g} / \mathrm{M}$; and in agar, the galactan backbone contains often impurities such as ester sulfate groups, methyl groups, or pyruvic acid acetal groups (Fig. 1)[1].

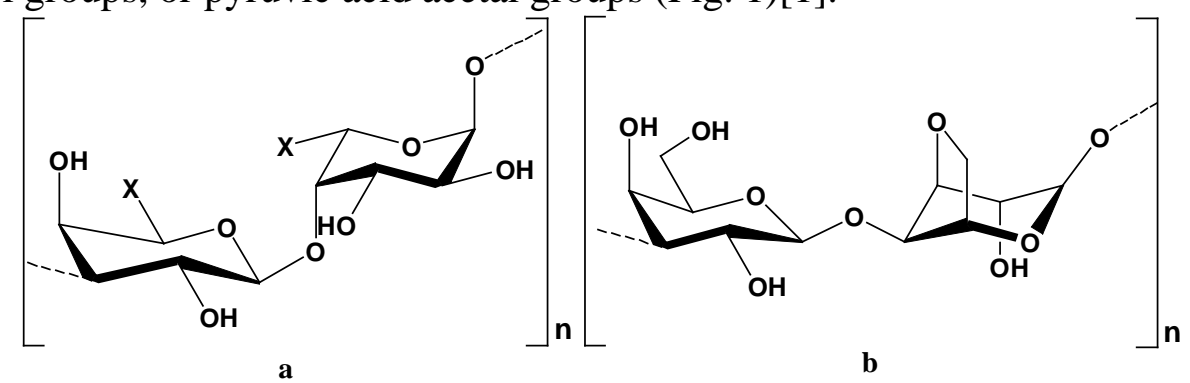

Fig. 1 Agar(a) and agarose(b) conformation in agar, X stands for ester sulfate groups, methyl groups, alcohol groups or pyruvic acid acetal groups.

In addition, agar showed an obvious advantage in the field of spinning because its favorable properties such as less toxicity, widely available and possibility for large-scale application. In the process of fiber forming solidification, the investigated ternary system is composed of polymer, solvent and nonsolvent, which may occur a variety of phase transition and the most important is phase separation, which occurs during the addition of a limited amount of nonsolvent into aqueous solution with certain mass fraction of polymer. The structure and performance of the fibers have close relation with the process of phase separation [2]. Hence, it is essential to investigate the phase behavior and separation mechanism of the polymer $(P)$-solvent $(S)$-nonsolvent $(N)$ ternary system for the purpose of designing a better spinning process. 
As a research of the mechanism of agar's forming process, this paper reports measurements on the phase behaviors of Agar-DMSO-EtOH system at different temperatures. Binodal curve was determined by cloud-point curve through the use of a titration method. A ternary phase diagram ranging from $25^{\circ} \mathrm{C}$ to $65^{\circ} \mathrm{C}$ was drawn to clearly show the effects of the temperature. In addition to Agar-DMSO-EtOH system, ternary phase diagram of Agar-DMSO- $\mathrm{H}_{2} \mathrm{O}$ system was also determined through the same method.

\section{Experimental}

\subsection{Sample preparation.}

Agar powder(Gel Strength(1.5\%):1200g/ $\mathrm{cm}^{2}$, Lan Ji production technology development co., LTD, Shanghai, China) is a biological reagent. weighing out a certain amount of agar power using an electronic analytical balance (ME104E, Mettler Toledo co., LTD, Shanghai, China) with a precision of $\pm 0.0001 \mathrm{~g}$.

Agar power was dispersed in $25 \mathrm{ml}$ of dimethyl sulfoxide (DMSO) and stirred at $95^{\circ} \mathrm{C}$ for 30 min to allow for complete dissolution. Then the Agar-DMSO solutions of a known concentration (2, 3, 4, 5, and $6 \%(\mathrm{w} / \mathrm{w}))$ were put into $50 \mathrm{ml}$ conical flasks, degassed at $25{ }^{\circ} \mathrm{C}$ for 2 hours. Until the Agar-DMSO solution becomes transparent, uniform and no bubble.

\subsection{Measurement.}

Titration experiment was measured in thermostat water bath(DF-101S, Qingdao lanteen science and education equipment co., LTD). Adjust the temperature to titrate temperature. Add the ethanol or distilled water drip into the Agar-DMSO solution drop by drop slowly, until the solution become cloudy and no longer clear in a minute after stirring, indicating the solution has reached turbidity. Record the volume of nonsolvent. Each reported value is the average of three consecutive readings.

The mass percent of the ternary systems of agar (P): DMSO (S): EtOH or $\mathrm{H}_{2} \mathrm{O}(\mathrm{N})$ could be determined through the following equation:

$$
m_{P}: m_{S}: m_{N}=m_{P}: \rho_{S} V_{S}: \rho_{N} V_{N}
$$

Where $P, S$ and $N$ are polymer, solvent and nonsolvent respectively, $m_{i}$ is the mass of ith component, $\rho_{i}$ is the density of $i$ th component, $V_{i}$ is the titration volume of $i$ th component.

Tab. 1 Data of cloud-point titration at different temperature

\begin{tabular}{ccccccc}
\hline $\begin{array}{c}C_{\text {agar }} \\
(\mathrm{w} / \mathrm{w})\end{array}$ & \multicolumn{3}{c}{$25^{\circ} \mathrm{C}$} & \multicolumn{3}{c}{$45^{\circ} \mathrm{C}$} \\
$(\%)$ & $\begin{array}{c}V_{\mathrm{EtOH}} \\
(\mathrm{ml})\end{array}$ & $m_{P}: m_{S}: m_{N}$ & $\begin{array}{c}V_{\mathrm{EtOH}} \\
(\mathrm{ml})\end{array}$ & $m_{P}: m_{S}: m_{N}$ & $\begin{array}{c}V_{\mathrm{EtOH}} \\
(\mathrm{ml})\end{array}$ & $m_{P}: m_{S}: m_{N}$ \\
\hline 2 & 24.22 & $1.18: 57.97: 40.85$ & 24.51 & $1.18: 57.69: 41.13$ & 24.96 & $1.17: 57.26: 41.57$ \\
3 & 23.78 & $1.79: 58.05: 40.16$ & 24.12 & $1.78: 57.72: 40.50$ & 24.61 & $1.77: 57.27: 40.98$ \\
4 & 23.48 & $2.42: 57.98: 39.60$ & 23.61 & $2.41: 57.85: 39.74$ & 24.08 & $2.39: 57.40: 40.21$ \\
5 & 23.12 & $3.05: 57.96: 38.99$ & 23.52 & $3.03: 57.58: 39.39$ & 24.02 & $3.00: 57.10: 39.90$ \\
6 & 23.06 & $3.68: 57.65: 38.67$ & 23.47 & $3.65: 57.25: 39.09$ & 23.95 & $3.62: 56.80: 39.57$ \\
\hline
\end{tabular}

\section{Results and discussion}

\subsection{Experimental cloud-point data analysis.}

There is no apparent turbidity when the agar concentration $\left(C_{\text {agar }}\right)$ is lower than $2 \%$, and the viscosity is too large to titrate when the concentration more than $6 \%$. So we chose concentration range of $2 \sim 6 \%$ that could actually carry out titration. Titration data listed in Tab. 1.

Table 1 shows the agar mass, ethanol volume and the mass percent of three components in end of titration at $25^{\circ} \mathrm{C}, 45^{\circ} \mathrm{C}$ and $65^{\circ} \mathrm{C}$. In Fig. 2, we can see ethanol volume $\left(V_{\mathrm{EtOH}}\right)$ decrease with the increasing of agar concentration $\left(C_{\text {agar }}\right)$. This is related to the intermolecular interaction of agar, which strengthened with the increase of $C_{\text {agar }}$, and further long-chain molecules interwoven with each other form a entanglement structure, which is conducive to its ability of gel. Moreover, temperature was another impact on the agar gelation: agar solution showed relatively low ability to gelate at 
higher temperature. This is mainly due to the enhanced molecular thermal motion that make it difficult to form a entanglement structure. Based on the above, we can draw a conclusion that increase concentration and lower temperature can help improve the ability of gelation.

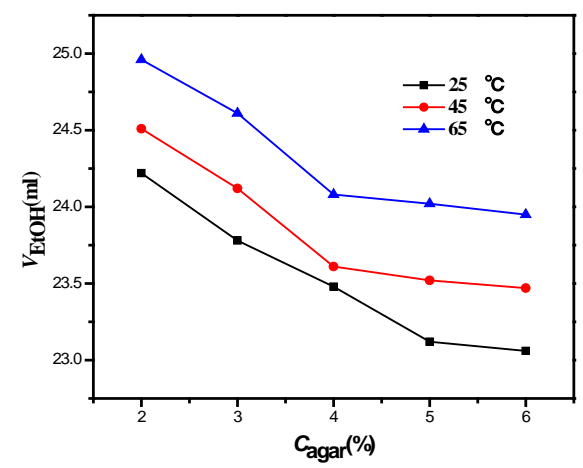

Fig. 2 Ethanol volume-agar concentration curves at different temperature.

\subsection{Cloud-point linear analysis}

Cloud-point curve was general considered approximate to the binodal curve by Boom etc. [3], as only occur liquid-liquid separation in titration experiment. So we can use cloud-point curve instead of binodal curve for analysis.

A linear relationship exists between the cloud points in $P-S-N$ ternary system under the different polymer concentration, and this relationship can be described by the following equation which derived from the Flory-Huggins Theory:

$$
\ln \frac{M_{N}}{M_{P}}=b \ln \frac{M_{S}}{M_{P}}+a
$$

Where $M_{\mathrm{i}}$ is the mass percent of ith component, $a$ and $b$ are constants which can be measured through experiment. Fig. 3 shows the $\ln \left(M_{\mathrm{EtOH}} / M_{\text {agar }}\right)$ and $\ln \left(M_{\mathrm{DMSO}} / M_{\text {agar }}\right)$ linear fitting of cloud point data.

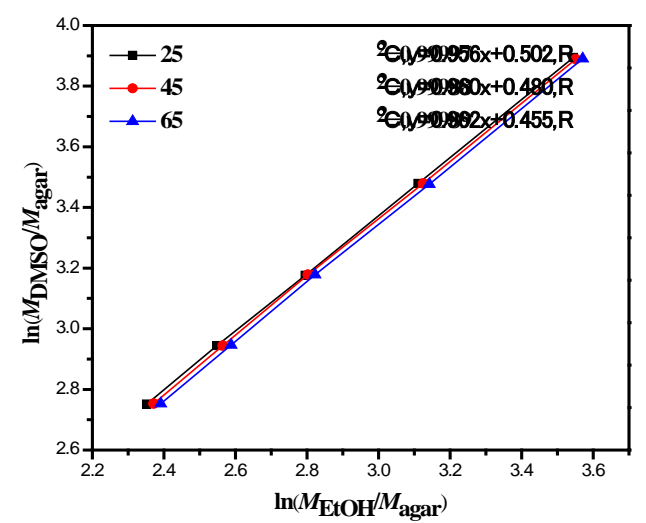

Fig. 3 The $\ln (\mathrm{MDMSO} / \mathrm{Magar})-\ln (\mathrm{MEtOH} / \mathrm{Magar})$ curves at different temperature.

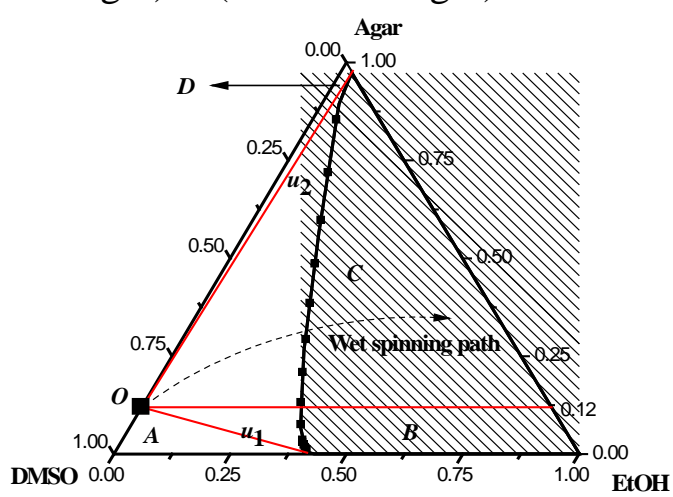

Fig. 4 The ternary phase diagram of Agar-DMSO-EtOH at $25^{\circ} \mathrm{C}$. u1-The first critical tangent, u2-The second critical tangent. 
As can be seen from Fig. 3, the linear correlation coefficients $\left(\mathrm{R}^{2}\right)$ of every fitting lines all exceed 99\% at different temperature, indicating a good linear relationship. According to LCP correlation of Agar-DMSO-EtOH system, we calculated the theoretical cloud-point data under the agar concentration of $12 \%, 20 \%, 30 \%, 40 \%, 50 \%, 60 \%, 70 \%, 80 \%$ and $90 \%$ at 25,45 and $65{ }^{\circ} \mathrm{C}$.

\subsection{Ternary phase diagram of Agar-DMSO-EtOH}

As shown in Fig. 4, diagram can be divided into two zones by cloud-point curve. The left zone is single phase region of ternary system that mixed with each other, the right shaded zone is multi phase region of ternary system in which polymer has solidified. O represents the spinning dope composition (Cagar=12\%). Starting from the point $\mathrm{O}$, three red lines which represent the spinning paths that are determined by the ratio of solvent transfer rate (JS) and nonsolvent transfer rate (JN) [4] can divide phase diagram into four areas, which are as following:

In area $\mathrm{A},-\infty \leq \mathrm{JS} / \mathrm{JN} \leq \mathrm{u} 1$, spinning dope was diluted continuously, the system was always in homogeneous and without solidification.

In area $\mathrm{B}, \mathrm{u} 1<\mathrm{JS} / \mathrm{JN} \leq 1$, spinning dope was also diluted, but phase separation occured when the concentration of nonsolvent reaches a certain value, then spinning dope solidified and form loosened structure.

In area $\mathrm{C}, 1<\mathrm{JS} / \mathrm{JN} \leq \mathrm{u} 2$, the content of polymer increased, the system occured phase separation and solidification. So the mechanical properties of the fibers obtained in this area are more homogeneous than area $\mathrm{B}$.

In area $\mathrm{D}, \mathrm{u} 2<\mathrm{JS} / \mathrm{JN} \leq \infty$, including gellization for spinning dope and orientational crystallization for lyotropic liquid crystal polymer(LLCP). Fibers formed under this condition are the best in quality.

Usually wet spinning paths distributed in area $\boldsymbol{C}$ and accompanied with phase separation that appeared when the wet spinning path intersects with cloud-point curve. The spinning dope composition and wet spinning path determine the structure of as-spun fibers. Therefore, we can adjust the spinning conditions such as dope concentration, the concentration and temperature of coagulation bath to obtain as-spun fibers that have dense and uniform structure.

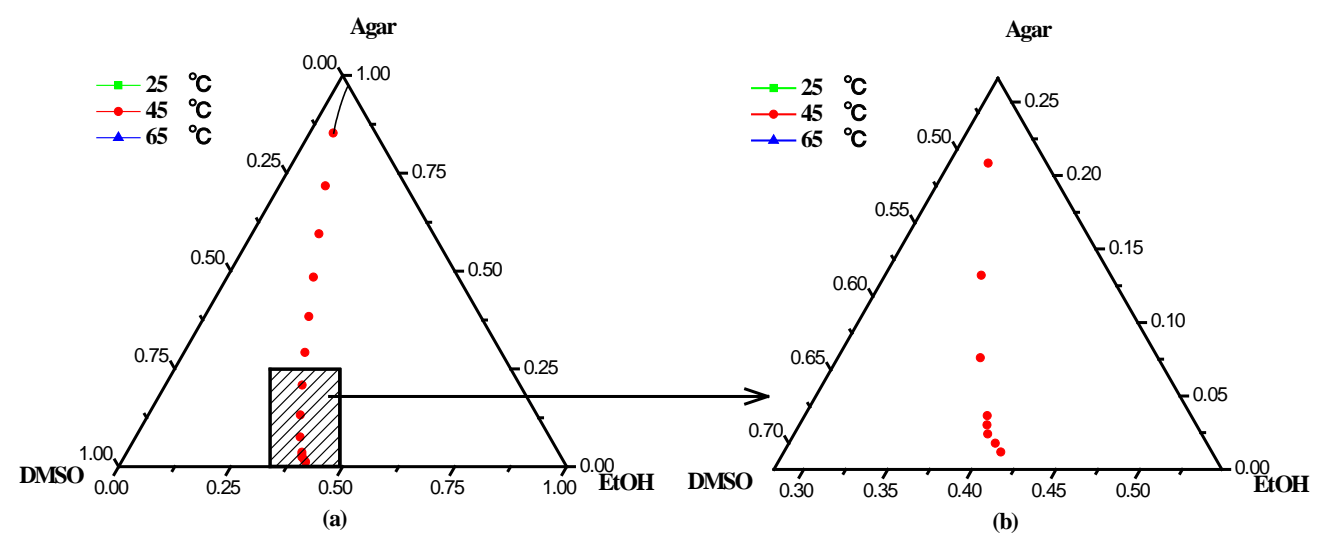

Fig. 5 The ternary component phase diagrams of Agar-DMSO-EtOH at different temperature.

(a)-original diagram, (b)-local enlargement diagram

Fig. 5 shows the cloud-point curves of Agar-DMSO-EtOH at $25^{\circ} \mathrm{C}, 45^{\circ} \mathrm{C}$ and $65^{\circ} \mathrm{C}$. Here, cloud-point curves that deviated from the Agar-DMSO axis with temperatures rise can be regarded as binodal curves. As shown in the figure, as temperatures rise, binodal curves moving away from the Agar-DMSO axis. This is due to the solidification ability of EtOH for agar descend with the rise of temperature. Otherwise, the rise in temperature make agar easy to dissolve in DMSO. The closer the binodal curve and Agar-DMSO axis the less EtOH needed in the process of solidifying. And got as-spun fibers with higher solid content. As a consequence, low temperature was beneficial to produce fibers with dense structure and fewer holes.

Compared with the Agar-DMSO-EtOH system, the Agar-DMSO- $\mathrm{H}_{2} \mathrm{O}$ system has advantages of having a low cost and less toxicity. But for the Agar-DMSO- $\mathrm{H}_{2} \mathrm{O}$ system, the cloud-point 
phenomenon was not obvious because of the agar can rapidly form into hydrogel when meeting water, which makes the phase separation difficult to observe. We got the rough ternary component phase diagram of Agar-DMSO- $\mathrm{H}_{2} \mathrm{O}$ using the same way as above. The cloud-point curve of Agar-DMSO- $\mathrm{H}_{2} \mathrm{O}$ showed in Fig. 6.

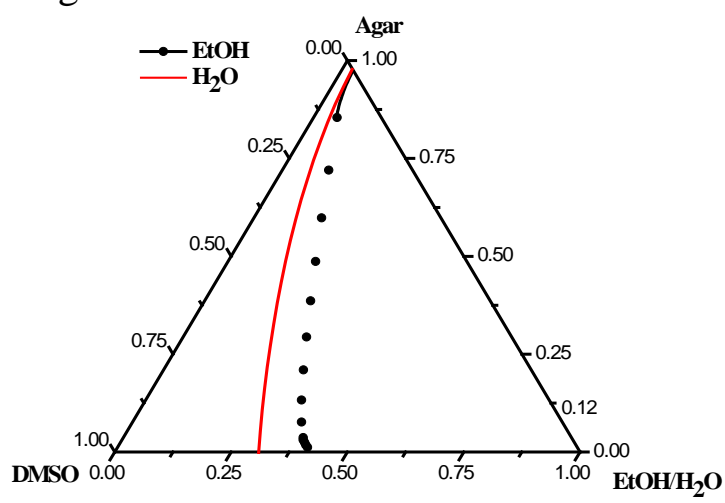

Fig. 6 The comparison of Agar-DMSO-EtOH and Agar-DMSO-H2O ternary component phase diagram at $25^{\circ} \mathrm{C}$.

As can be seen from the Fig. 6, water is slightly better than ethyl alcohol to solidify agar. This indicates that water as coagulation bath on agar spinning is feasible. And fibers that have good properties has been obtained in actual spinning by using water as coagulating bath. But because of the formation of hydrogel, the strength of as-spun fibers from water were worse than that from ethyl alcohol.

\section{Conclusions}

We can obtain as-spun dense and uniform fibers by adjusting the dope concentration, the concentration and temperature of coagulation bath. High concentration and low temperature are contribute to improve agar's ability of gelation. Low temperature was beneficial to produce fibers with dense structure and fewer holes. Instead of ethyl alcohol with water as coagulation bath in agar spinning is feasible.

\section{References}

[1]. Mickael Cregut, Emmanuel Rondags. New insights in agar biorefinery with arylsulphatase activities[J]. Process Biochemistry, 2013, 48, 12:1861-1871.

[2]. Ziabicki A. Fundamentals of fibre formation[M]. Shanghai Science and Technology Press. 1993, 138-142

[3]. Boom R M, Boomgaard V D, Smolders C A, Linearized cloud-point curve correlation for ternary systems consisting of one polymer, one solvent and one non-solvent[J]. Polymer, 1993, 34(11): 2348-2356.

[4]. Xinyuan Shen. Polymer materials processing principle[M]. China TeXtile Press, 2007, 213-214. 